\title{
The binding of a monoclonal antibody to the apical region of SCARB2 blocks EV71 infection
}

\author{
Xuyuan Zhang ${ }^{1,3}$, Pan Yang ${ }^{2,3}$, Nan Wang ${ }^{2,3}$, Jialong Zhang ${ }^{1}$, Jingyun $\mathrm{Li}^{1}$, Hao Guo ${ }^{1}$, Xiangyun Yin ${ }^{1}$, \\ Zihe Rao ${ }^{2}$, Xiangxi Wang ${ }^{2 \bowtie}$, Liguo Zhang ${ }^{1 凶}$ \\ ${ }^{1}$ Key Laboratory of Infection and Immunity, Institute of Biophysics, Chinese Academy of Sciences, Beijing 100101, China \\ ${ }^{2}$ National Laboratory of Macromolecules, Institute of Biophysics, Chinese Academy of Sciences, Beijing 100101, China \\ ${ }^{3}$ University of Chinese Academy of Sciences, Beijing 100049, China \\ $\square$ Correspondence: xiangxi@ibp.ac.cn (X. Wang), liguozhang@sun5.ibp.ac.cn (L. Zhang) \\ Received February 22, 2017 Accepted March 16, 2017
}

\begin{abstract}
Entero virus 71 (EV71) causes hand, foot, and mouth disease (HFMD) and occasionally leads to severe neurological complications and even death. Scavenger receptor class B member 2 (SCARB2) is a functional receptor for EV71, that mediates viral attachment, internalization, and uncoating. However, the exact binding site of EV71 on SCARB2 is unknown. In this study, we generated a monoclonal antibody $(\mathrm{mAb})$ that binds to human but not mouse SCARB2. It is named JL2, and it can effectively inhibit EV71 infection of target cells. Using a set of chimeras of human and mouse SCARB2, we identified that the region containing residues 77-113 of human SCARB2 contributes significantly to JL2 binding. The structure of the SCARB2-JL2 complex revealed that JL2 binds to the apical region of SCARB2 involving a-helices 2,5 , and 14 . Our results provide new insights into the potential binding sites for EV71 on SCARB2 and the molecular mechanism of EV71 entry.
\end{abstract}

KEYWORDS SCARB2, EV71, monoclonal antibody, HFMD, receptor

Xuyuan Zhang and Pan Yang contributed equally to this work.

Electronic supplementary material The online version of this article (doi:10.1007/s13238-017-0405-7) contains supplementary material, which is available to authorized users.

\section{INTRODUCTION}

Hand, foot, and mouth disease (HFMD) is a common viral illness that usually affects infants and children younger than 5 years old (Ooi et al., 2010). Both Entero virus 71 (EV71) and Coxsackie A virus type 16 (CA16) are common causative agents (Fan et al., 2013; Kim et al., 2013; Zou et al., 2012). HFMD is often mild and self-limiting. However, unlike CA16, EV71 infection occasionally causes acute encephalitis, acute flaccid paralysis, and cardiopulmonary failure. EV71-associated neurological complications sometimes can be fatal ( $\mathrm{Ho}$ et al., 1999; McMinn, 2002; Yamayoshi et al., 2012). In recent years, an increasing number of reports on HFMD outbreaks with fatal cases because of EV71 infection in China (Liu et al., 2011; Wang et al., 2015; Zhang et al., 2014; Zhou et al., 2013), Australia (Sanders et al., 2006), Singapore (Chan et al., 2003; Wu et al., 2010), Malaysia (Chua et al., 2007; Chua and Kasri, 2011; Ooi et al., 2007), Korea (Cho et al., 2010; Kim et al., 2013; Lee et al., 2016), and Japan (Hosoya et al., 2006; Mizuta et al., 2014; Sato et al., 2006) have been reported. Thus, EV71 infection is a serious public health problem across the AsianPacific region.

Human scavenger receptor class B member 2 (SCARB2; also known as lysosomal integral membrane protein II or LGP85) (Yamayoshi et al., 2009) has been identified as the functional cellular receptor for EV71. SCARB2 is a type III transmembrane protein that belongs to the scavenger receptor family (Vega et al., 1991a; Vega et al., 1991b). It is widely expressed on various cell types, including neurons (Yamayoshi et al., 2014). SCARB2 can serve as a receptor for all tested EV71 strains (Yamayoshi et al., 2013). SCARB2 mediates EV71 attachment and internalization through the 
clathrin-mediated endocytic pathway (Lin et al., 2012), and it is essential for EV71 uncoating at low pH (Dang et al., 2014; Yamayoshi et al., 2013). Transgenic mice with human SCARB2 overexpression are susceptible to EV71 infection (Fujii et al., 2013; Lin et al., 2013; Zhou et al., 2016). Thus, SCARB2 plays a critical role in EV71 infection and pathogenesis.

SCARB2 has a twisted $\beta$-barrel core, and $\alpha$-helices 4,5 , and 7 form a three-helix bundle that is the possible interaction site for its ligand (Neculai et al., 2013). Because mouse SCARB2 is not an efficient EV71 receptor, it is possible to identify the virus-binding site using chimeras of mouse and human SCARB2. Human SCARB2 amino acid residues 142-204 are important for EV71 binding and infection (Yamayoshi and Koike, 2011). Additionally, the critical amino acids for SCARB2 binding to EV71 were further mapped to residues 144-151, which is a highly variable region (HVR) among species (Chen et al., 2012). Soon afterwards, Dang et al. demonstrated the residues 146-166 are also essential for EV71 attachment (Dang et al., 2014). All the mapped binding sites are mainly located in the three-helix bundle of $\alpha 4, \alpha 5$, and $\alpha 7$. However, there is no direct evidence that identifies the binding sites of EV71 on SCARB2, since the complex structure of EV71-SCARB2 is not available.

Until now, there have been no reports of a monoclonal antibody (mAb) of SCARB2 that could block EV71 infection. In the current work, we characterized a mAb against human SCARB2, called JL2, which is capable of blocking EV71 infection in vitro. The complex structure of SCARB2-JL2 and a chimeric binding assay demonstrated that JL2 interacts with a-helices 2, 5, and 14 in human SCARB2. Our results provide additional support for the recognition sites of EV71 on SCARB2.

\section{RESULTS}

\section{Characterization of an anti-SCARB2 mAb, JL2}

A mAb against human SCARB2, JL2, was produced with conventional procedures as described in the "MATERIALS AND METHODS" section. JL2 was purified using Protein G from ascites and tested by SDS-PAGE. The purity of the purified JL2 was greater than $96 \%$. We also analyzed the heavy chain and light chain of JL2 by FACS staining and an ELISA and found that JL2 is formed by the $\lg$ G2a heavy chain and the $\mathrm{K}$ light chain (data not shown).

To confirm that JL2 recognizes human SCARB2 specifically, we established a 293T cell line with the SCARB2 gene knocked out (293-SCARB2-KO) by using CRISPR-CAS9 technology. We also established the stable cell line 293-hSCARB2 by transducing 293A cells with a lentiviral vector with the coding sequence for human SCARB2. Then, we used JL2 to stain the cells lysates from the above-mentioned cell lines. As shown in Figure 1A, JL2 could stain SCARB2 (85 kDa) in the overexpressing cell line but not SCARB2-KO cells. In the 293-hSCARB2 cells, which stably expressed human SCARB2 on the cell surface, we showed that JL2 could bind to SCARB2 without permeabilization (Fig. 1B). Thus, JL2 could bind to human SCARB2 on the plasma membrane. With serial dilution of JL2, we showed that the binding of JL2 to 293-hSCARB2 increased from $0.01 \mu \mathrm{g} / \mathrm{mL}$ and plateaued at a concentration of $2 \mu \mathrm{g} / \mathrm{mL}$ (Fig. 1C). Collectively, JL2 is a human SCARB2 specific $\mathrm{mAb}$ that can bind to SCARB2 on the cell surface.

\section{JL2 inhibits EV71 infection}

To facilitate the analysis of EV71 infection, we constructed an infectious clone with the coding sequence for EGFP inserted between the 5'NTR and VP4 of the EV71 genome (EV71-GFP), as described previously (Yamayoshi et al, 2009). As shown in Figure S1, EV71-GFP-infected 293-hSCARB2 cells express GFP and can be visualized under a fluorescence microscope.

To analyze the inhibitory effect of JL2 on EV71 infection, we pretreated the 293-hSCARB2 cells with JL2 for $1 \mathrm{~h}$ and then incubated with EV71-GFP virus at $0.1 \mathrm{MOI}$. As a result, the ratio of GFP-positive cells decreased dramatically, and the cytopathic effect (CPE) was inhibited by JL2 but not the isotype control antibody (Fig. S2). Additionally, JL2 inhibited EV71 infection in a dose-dependent manner, and the inhibitory effect could be observed at concentrations as low as $0.1 \mu \mathrm{g} / \mathrm{mL}$ (Fig. 2A).

Accordingly, the production of infectious EV71 was also blocked by the JL2 mAb. We pretreated 293-hSCARB2 cells with JL2 or IgG2a. Then, we infected the pretreated cells with wild type EV71 (at MOI of 0.1 ). After $18 \mathrm{~h}$ of infection, we quantified the viral RNA by real-time PCR. Consistent with EV71-GFP infection, JL2 also inhibited the wild type EV71 infection of 293-hSCARB2 cells (Fig. 2B).

Taken together, these results demonstrated that JL2 mAb effectively inhibits EV71 infection.

Identification of the binding site of JL2 on human SCARB2

JL2 binds to human SCARB2 but not mouse SCARB2 (Fig. S3) but the commercial polyclonal antibody Human LIMPII/SR-B2 Antibody (AF1966) from R\&D Systems could bind to both at a similar level (data not shown). We compared the sequences of mouse and human SCARB2 (Fig. 3A) and highlighted the apical domains on the top of the SCARB2 molecules (Fig. 3A and 3B), that potentially bind to EV71 and facilitate its entry and infection (Canton et al., 2013; Dang et al., 2014; Gao et al., 2014). Based on the sequence analysis, we constructed a serious of chimeras of human and mouse SCARB2 (Fig. 3C) to map the binding site of JL2. We found that the residues 1-76 of human SCARB2 do not contribute to JL2 binding (Fig. 3C and 3D). However, JL2 binds to a chimera including residues 1-113 
A

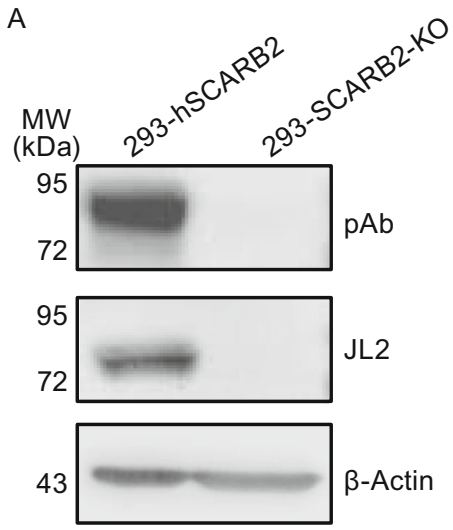

C

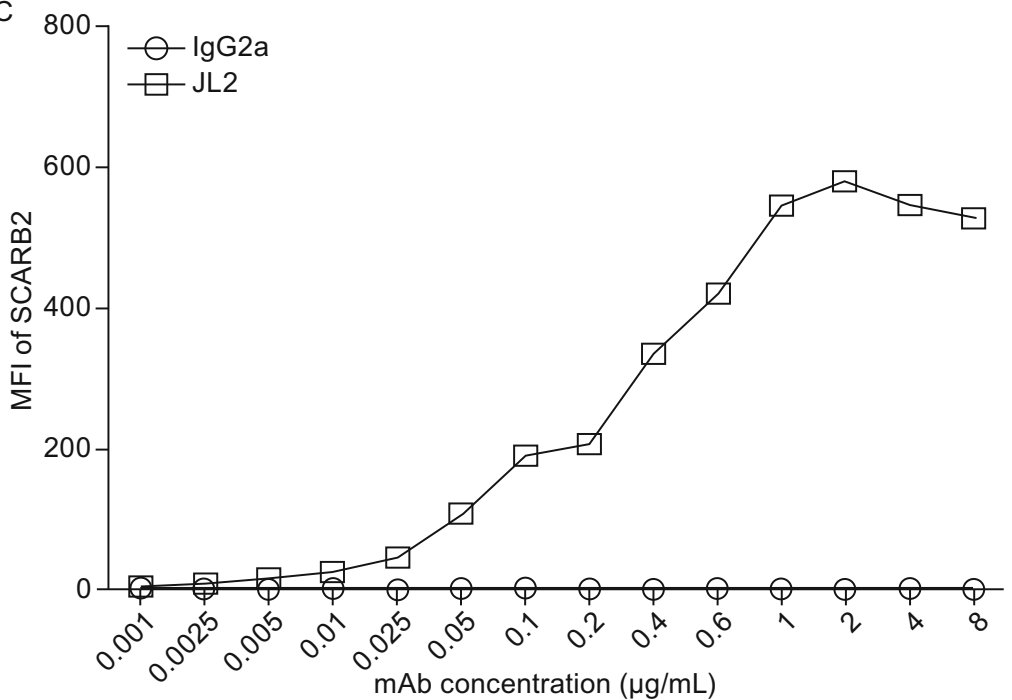

B

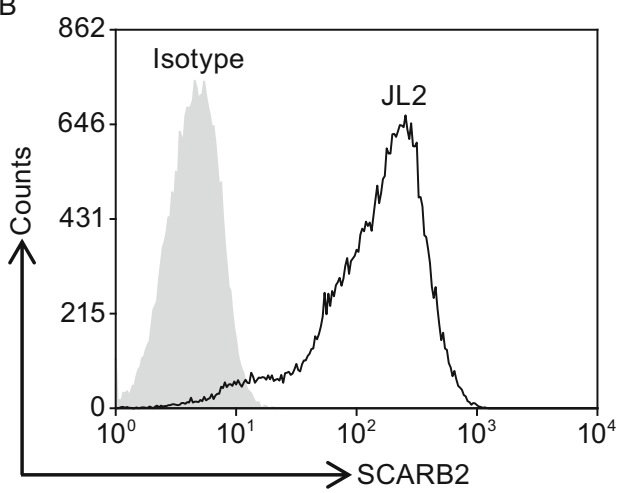

Figure 1. The monoclonal antibody JL2 binds human SCARB2. (A) Cells overexpressing human SCARB2 and SCARB2 knockout cells were lysed and analyzed by Western blot using the anti-human SCARB2 polyclonal antibody (pAb) (top panel) and JL2 (middle panel). The lowest panel: $\beta$-actin was used as a loading control. (B) Surface staining of 293-hSCARB2 cells. Open area represents staining with JL2 and shaded area represents isotype control staining. (C) The binding of 293-hSCARB2 cells with different concentrations of JL2 (square) and the IgG2a isotype control (circle).

A

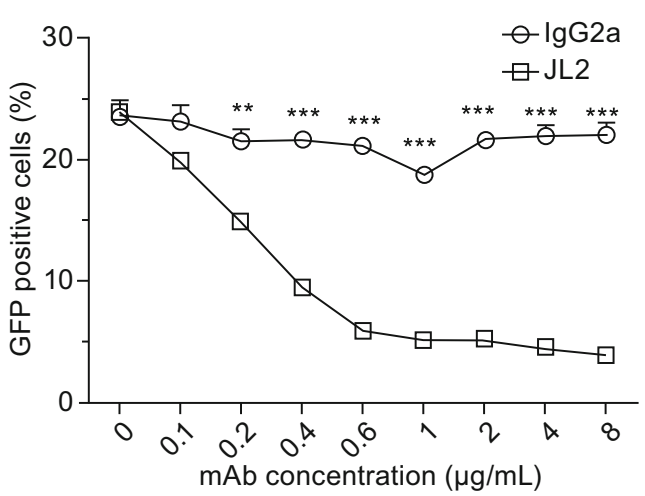

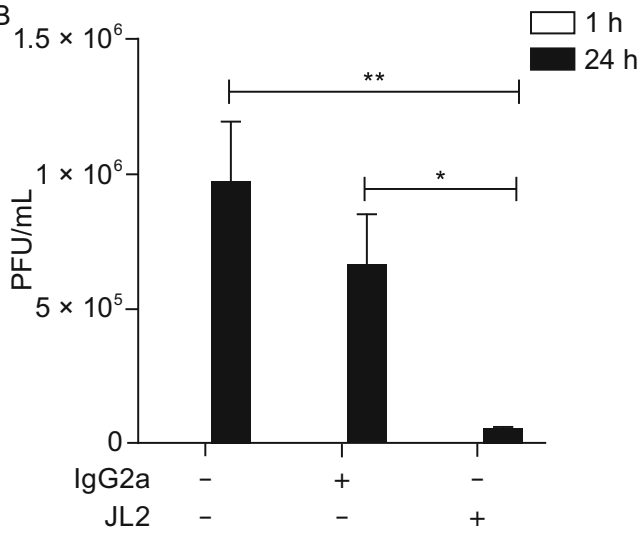

Figure 2. JL2 blocks EV71 infection. (A) GFP-positive cell counts among 293-hSCARB2 cells pretreated with JL2 (square) or the isotype control (circle) before EV71-GFP infection. The data are shown as the mean \pm SEM of triplicates. (B) The replication of wild type EV71 in 293-hSCARB2 cells in the presence of $\mathrm{JL} 2(2 \mu \mathrm{g} / \mathrm{mL})$ or isotype control. The data are presented as the mean \pm SEM of triplicates. ${ }^{*} P<0.05,{ }^{* *} P<0.01$, and ${ }^{* \star *} P<0.001$ by Student's $t$ test. 
A

Human (1) ${ }^{1}$ MGRCCFYTAGTLSLLLLVTSVTLLVARVFQKAVDQSIEKKIVLRNGTEAFDSWEKPPLPY

Mouse (1) MGRCCFYTAGTLSLLLLVTSVTLLVARVFQKAVDQT IEKNIVLQNGTKVFNSWEKPPLPY

$\begin{array}{lll}61 & \alpha 2 & 120\end{array}$

Human (61) YTQFYFFNVTNPEEILRGETPRVEEVGPYTYRELRNKANIQFGDNGTTISAVSNKAYVFE

Mouse (61) YIQFYFFNVTNPEEILQGEIPLLEEVGPYTYRELRNKANIQFGENGTTISAVTNKAYVFE 121 a4 a5

180

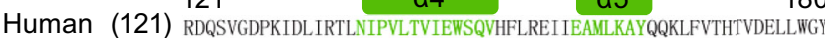
Mouse (121) RVQSVGDPNVDLIRT INIPLLTVVDLAQLTLLRELIEAMLKAYQQKLFVIHTVHELLWGY

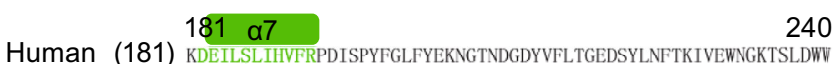
Mouse (181) KDEILSLVHIFKPDVSPNFGLFYERNGTNDGEYVFLTGEDNYLNFSKIVEWNGKTSLDWV

Human (241) $\begin{array}{lr}241 & 300 \\ \text { ITDKCNMINGTDGDSFHPLITKDEVLYVFPSDFCRSVYITFSDYESVQGLPAFRYKVPAE }\end{array}$ Mouse (241) TTDTCNMINGTDGDSFHPLISKDEVLYLFPSDLCRSVHITFSSFENVEGLPAFRYKVPAE 301

Human (301) ILANTSDNAGFCIPEGYCLGSGVLNYSICKNGAPIIMSFPHFYQADERTIS 360 Mouse (301) ILANTSENAGFCIPEGNCMDSGVLNISICKNGAPIIMSFPHFYQADEKFVSATKGMHPNE 361

a14

Human (361) EDHETFVDINPLTGIILKAAKRFQINIYVKKLDDFVETGDIRTMVFPVMYLNESVHIDKE Mouse (361) EEHESFVDINPLTGIILRGAKRFQINTYVRKLDDFVETGDIRTMVFPVMYLNESVLIDKE

$$
421
$$

Human (421) TASRLKSMINTTLIITNIPYITMALGVFFGLVFTWLACKGgGSMDEGTADERAPLIRT Mouse (421) TANQLKSVINTTLVVTNIPYIIMALGVFFGLVFTWLACRGQGSMDEGTADERAPLIRT

C

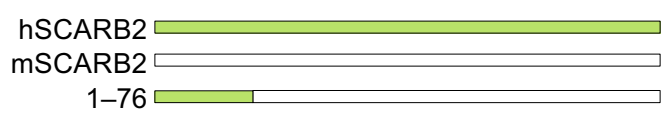

$77-478$

$1-113$

$114-478$

$1-166$

$167-478$

1-301

302-478

$144-151$

144-151\&302-478

1-166\&302-478
D

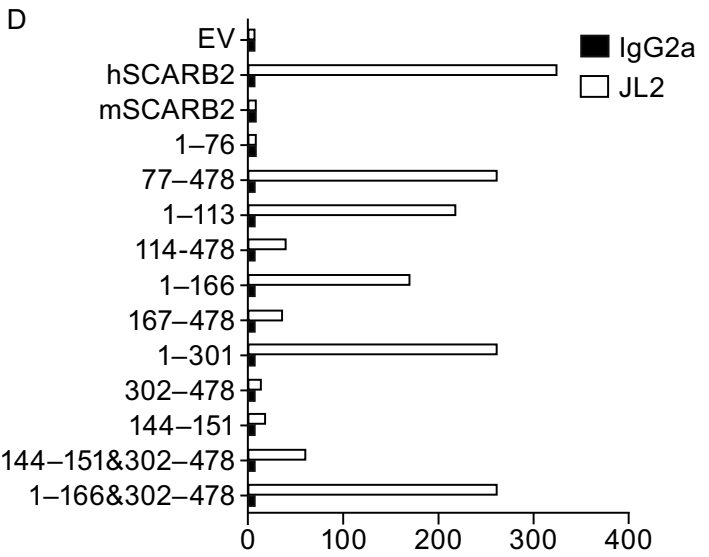

B

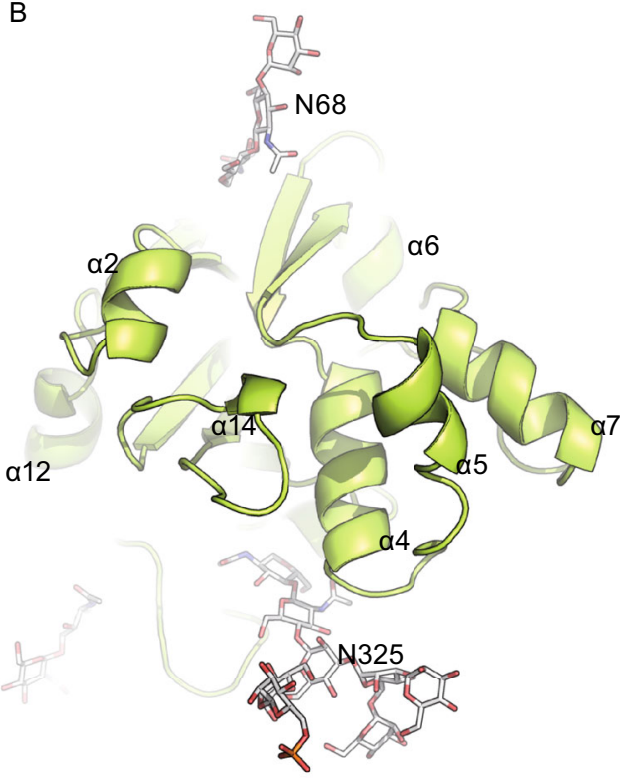

Figure 3. Binding site mapping of JL2 and human SCARB2. (A) An alignment of the mouse and human SCARB2 with the sequences of the 5 a-helices in the apical region highlighted. (B) The structure of the 5 a-helices in the apical region of human SCARB2 at neutral $\mathrm{pH}$. (C) The sketch map of the SCARB2 chimeras. The numbers represent the amino acid sequences of human SCARB2 in the chimeras. (D) 293-SCARB2-KO cells transfected with SCARB2 chimeras were stained by JL2 (open bars) or the isotype control (filled bars). The data shown are representative of 3 independent experiments.

from human SCARB2, suggesting that the residues 77-113 contribute significantly to JL2 binding (Fig. $3 \mathrm{C}$ and $3 \mathrm{D}$ ). The apical helices of SCARB2, including $\alpha 4$ and $a 5$, have been reported to contribute to the preferential EV71 binding of human SCARB2 over its mouse counterpart (Chen et al., 2012; Dang et al., 2014). However, chimeric SCARB2 with the residues 1-166 from the human sequence did not show a stronger binding affinity than a chimera only containing the residues 1-113. JL2 did not bind the chimera with the residues $302-478$ from human SCARB2, but it did bind the chimera with the combination of the residues 144-151 and 302-478.

Collectively, we found that there are at least three regions of human SCARB2, residues 77-113, 144-151, and 302478 , those contribute to the binding of JL2 mAb.

\section{Structure determination}

To dissect the molecular interaction mechanism of human SCARB2 and JL2 directly, we sequenced the CDNA of both the light chain and heavy chain of JL2, from the sequence, we found three common complementary determining regions (CDRs) in the heavy chain HCDR1 (residues 26-32), HCDR2 (residues 51-58), HCDR3 (residues 97-111) and three CDRs in the light chain LCDR1 (residues 28-33), LCDR2 (residues 51-53), LCDR3 (residues 90-98). The mAb sequence is necessary for us to analyze the SCARB2JL2 complex structure (Fig. S4). Meanwhile, the human SCARB2 luminal domain containing residues 27-429 was produced by the Bac-to-Bac expression system in Sf9 cells, and the JL2 Fab fragments were prepared using the Pierce 
FAB preparation kit (Thermo Scientific). The purified JL2 Fab fragments were incubated with purified SCARB2 at $4^{\circ} \mathrm{C}$ for $1 \mathrm{~h}$ at molar ratio of $1: 1$, following the further purification by size-exclusion chromatography (GE Healthcare). Crystals of SCARB2-JL2 complex diffracted to $3.5 \AA$, belonging to the space group $\mathrm{C} 2$, and the crystals contained two complex molecules in the asymmetric unit with a solvent content of $58 \%$ (corresponding to a Matthews coefficient $\mathrm{VM}=2.7$ $\AA^{3} \cdot \mathrm{Da}^{-1}$ ) (Matthews, 1968). The complex structure was determined using the molecular replacement method based on the combination of the models of SCARB2 at neutral pH (PDB code: 4TW2) (Dang et al., 2014) and the mouse Fab (PDB code: 5WTG) (Wang et al., 2017). The final refined complex model had reasonable $\mathrm{R}$-factors and very good stereochemistry. Details of the protein expression, purification, crystallization and structure determination are given in the "MATERIALS AND METHODS" section and Table 1.

\section{Complex structure of SCARB2 with JL2}

The complex structure revealed that JL2 binds to the head of SCARB2 (Domain III) at an approximately perpendicular angle via the three helices of $\alpha 2$, $\alpha 5$, and $\alpha 14$ (Fig. 4A). In particular, $\alpha 14$ inserts into the cavity formed by two common complementary determining regions (CDRs) in the heavy chain (Figs. 4B and 5). The helix $\alpha 2$ interacts with both the heavy and light chains. Although the helices $\alpha 4, \alpha 5$, and $\alpha 7$ form a three-helix bundle, only $\alpha 5$ is involved in the binding of human SCARB2. It is noteworthy that a5 undergoes a pivotal $\mathrm{pH}$-dependent conformational change to trigger viral uncoating (Dang et al., 2014) and in the complex model, SCARB2 still keeps the neutral conformation at $\mathrm{pH} 7.0$ (Fig. S5) and exhibits no notable conformational changes upon binding to JL2 Fab at neutral pH (the superimposition of helical bundle $\alpha 4, \alpha 5$, and $\alpha 7$ of SCARB2 in the complex with SCARB2 at $\mathrm{pH} 4.8(\mathrm{RMSD}=0.66), \mathrm{pH} 6.5(\mathrm{RMSD}=$ $0.42), \mathrm{pH} 7.5(\mathrm{RMSD}=0.41)$ ). This allows us to speculate that JL2 not only is able to prevent EV71 binding to SCARB2 but also locks the configuration of SCARB2 wherever it is under neutral or acidic environments.

\section{The amino acids in JL2 contributing to SCARB2 binding}

The heavy chain and light chain variable domains contribute approximately $74 \%$ (interaction area $625 \AA^{2}$ ) and approximately $26 \%$ (interaction area $225 \AA^{2}$ ) of the protein-protein interface, respectively, with the heavy chain binding the three helices $\alpha 2$, $\alpha 5$ and $\alpha 14$, while the light chain predominately binds $\alpha 2$ (Fig. 4A and 4B). The interaction surface on JL2 has five of the six common complementary determining regions (CDRs): HCDR1 (residues 28-32), HCDR2 (residues 54-55), HCDR3 (residues 100-107), LCDR1 (residues 31-32), and LCDR2 (residues 50-55) (Figs. 4C and 5). The epitopes on SCARB2 include residues E73, E74, L76, R77, and E79 in a2; K161 and Q164 in a5; K391, L392, D393, D394, F395, V396, E397, T398, and G399 in a14 (Figs. 4B,
Table 1. Data collection and refinement statistics

\begin{tabular}{|c|c|}
\hline Name & SCARB2-fab \\
\hline \multicolumn{2}{|l|}{ Data collection } \\
\hline Resolution $(\AA)$ & $50.00-3.50(3.63-3.50)$ \\
\hline Unique reflections & $30,522(3,025)$ \\
\hline Space group & C2 \\
\hline \multicolumn{2}{|l|}{ Cell dimensions } \\
\hline$a(\AA)$ & 199.9 \\
\hline$b(\AA)$ & 75.6 \\
\hline$c(\AA)$ & 164.3 \\
\hline$\alpha\left({ }^{\circ}\right)$ & 90.00 \\
\hline$\beta\left({ }^{\circ}\right)$ & 100.11 \\
\hline$V\left({ }^{\circ}\right)$ & 90.00 \\
\hline Redundancy & $2.5(2.5)$ \\
\hline Completeness (\%) & $99.7(99.8)$ \\
\hline Rmerge & $0.238(0.713)$ \\
\hline$\| / \sigma(I)$ & $9.67(2.10)$ \\
\hline \multicolumn{2}{|l|}{ Refinement } \\
\hline Resolution $(\AA)$ & 3.50 \\
\hline No. reflections & 30,508 \\
\hline$R_{\text {work }} / R_{\text {free }}$ & $0.231 / 0.294$ \\
\hline \multicolumn{2}{|l|}{ No. of non-H atoms } \\
\hline Protein & 12,756 \\
\hline Glycans & 562 \\
\hline Mean B-factor $\left(\AA^{2}\right)$ & 88.1 \\
\hline \multicolumn{2}{|c|}{ Ramachandran statistics (\%) } \\
\hline Most favored & 90.5 \\
\hline Allowed & 8.7 \\
\hline Outliers & 0.8 \\
\hline \multicolumn{2}{|l|}{ R.m.s.deviations } \\
\hline Bond lengths $(\AA)$ & 0.007 \\
\hline Bond angles $\left({ }^{\circ}\right)$ & 1.220 \\
\hline
\end{tabular}

Values in parentheses are for highest-resolution shell.

a $R_{\text {merge }}=\Sigma_{\text {hkl }} \Sigma_{\mathrm{i}}\left|/(\mathrm{hkl})_{\mathrm{i}}-</(\mathrm{hkl})>\right| / \Sigma_{\mathrm{hkl}} \Sigma_{\mathrm{i}}((\mathrm{hkl})$ i.

b $R_{\text {work }}=\Sigma_{\text {hkl }}\left|F_{\mathrm{o}}(\mathrm{hkl})-F_{\mathrm{c}}(\mathrm{hkl})\right| / \Sigma_{\text {hkl }} F_{\mathrm{o}}(\mathrm{hkl})$.

c $R_{\text {free }}$ was calculated for a test set of reflections (5\%) omitted from the refinement.

$5 \mathrm{~B}$, and Table 2). Tight binding is facilitated by 10 hydrogen bonds and a number of hydrophobic interactions. The antibody components of these interactions include residues D31, Y32, Y50, and 155 from the light chain and residues S28, T30, G31, Y32, Y54, Y55, R100, S102, Y105, and Y107 from the heavy chain (Figs. 4B, 5B, and Table 2). It is worth noting that the amino acids in $\alpha 5$ and $\alpha 14$ are identical between human and mouse SCARB2, and this agrees with the mapping data of the human and mouse SCARB2 
A

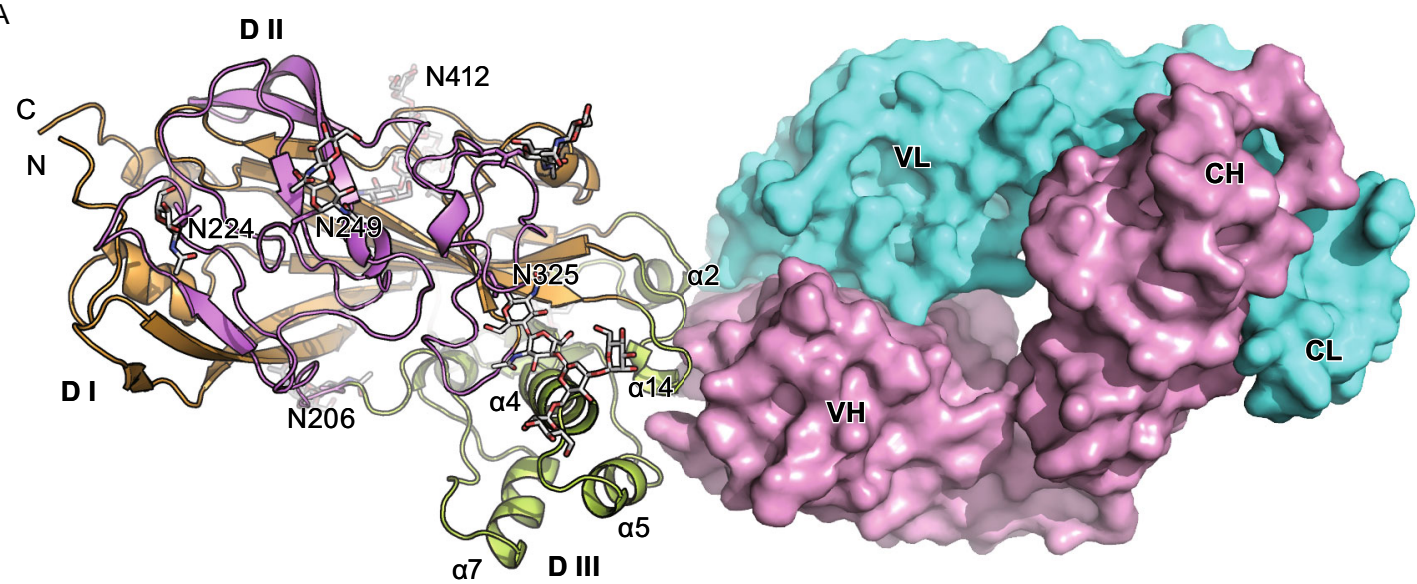

B

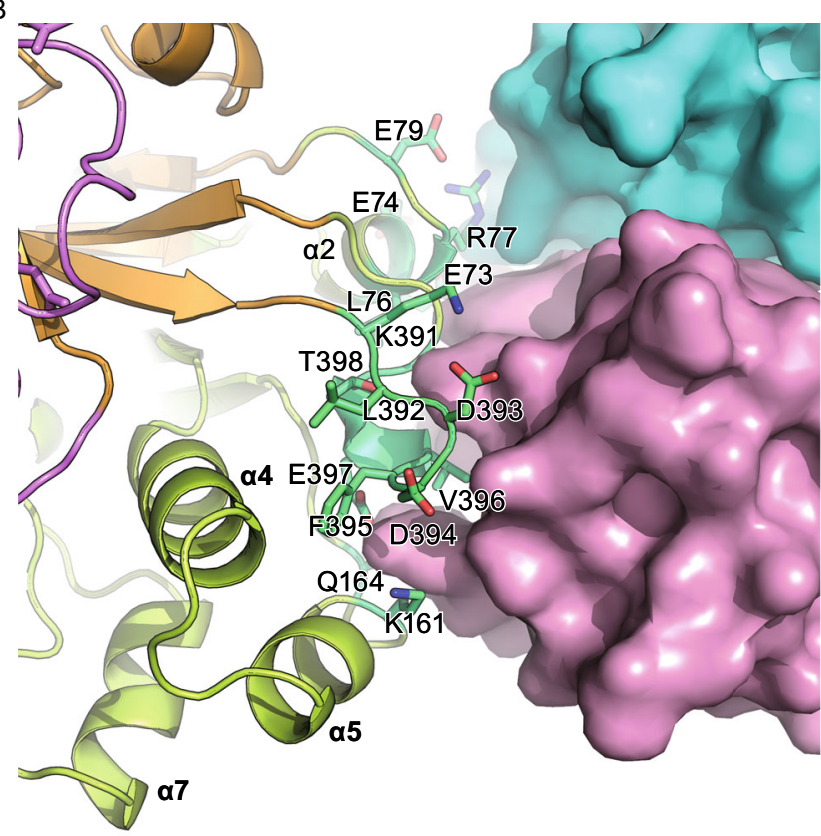

$c$

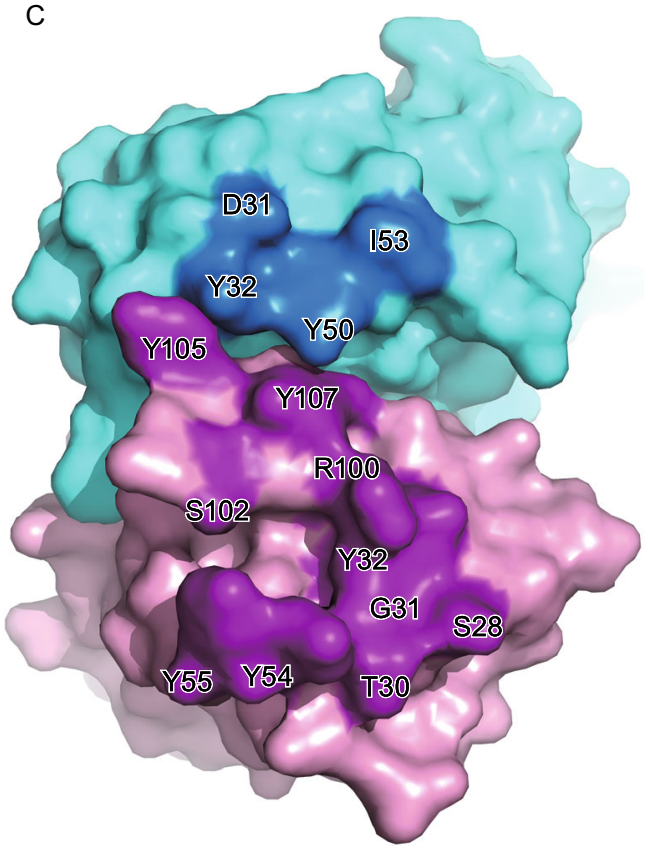

Figure 4. The complex structure of JL2 Fab bound to SCARB2 (at pH 7.0). (A) The overall complex structure. The JL2 Fab binds to the head of SCARB2 (Domain III) at a perpendicular angle with helices $\alpha 2$, $\alpha 5$, and $\alpha 14$ involved in the interaction. Domain I, II, and III of SCARB2 are colored in orange, violet and yellow, respectively. Glycans are shown as sticks. (B and C) Cartoon representation of the interacting residues in SCARB2 (B) and Fab (C). The residues in SCARB2 involved in the interactions with JL2 Fab are shown as sticks (B). The residues in JL2 Fab involved in the interactions with SCARB2 are highlighted in bright colors and labeled on the surface $(C)$.

chimeras. Even though, the residues $141-151$ of human SCARB2 contribute to the differential binding of JL2 to human and mouse SCARB2 (Fig. 3B), there is no direct interaction in the structure data. Thus, the residues 141-151 may contribute to the fold difference between human and mouse SCARB2, which affects JL2 binding.

\section{DISCUSSION}

In this report, we characterized a monoclonal antibody against human SCARB2, JL2, which blocks EV71 infection.
Structural modeling showed that JL2 binds to human SCARB2 via the three helices $\alpha 2$, $\alpha 5$, and $\alpha 14$ (Fig. 4A). JL2 binds to human SCARB2 but not mouse SCARB2. Among the 16 amino acids in human SCARB2 that contribute to JL2 binding, R77 is the only different residue between the human and mouse sequences (Q77). Thus, R77 may be the dominant contributor for the species specific binding of JL2 to human but not mouse SCARB2.

The helices $a 4$ and $a 5$ of human SCARB2 have been proposed to mediate EV71 binding and uncoating (Chen et al., 2012; Dang et al., 2014). The replacement 144-151 
A

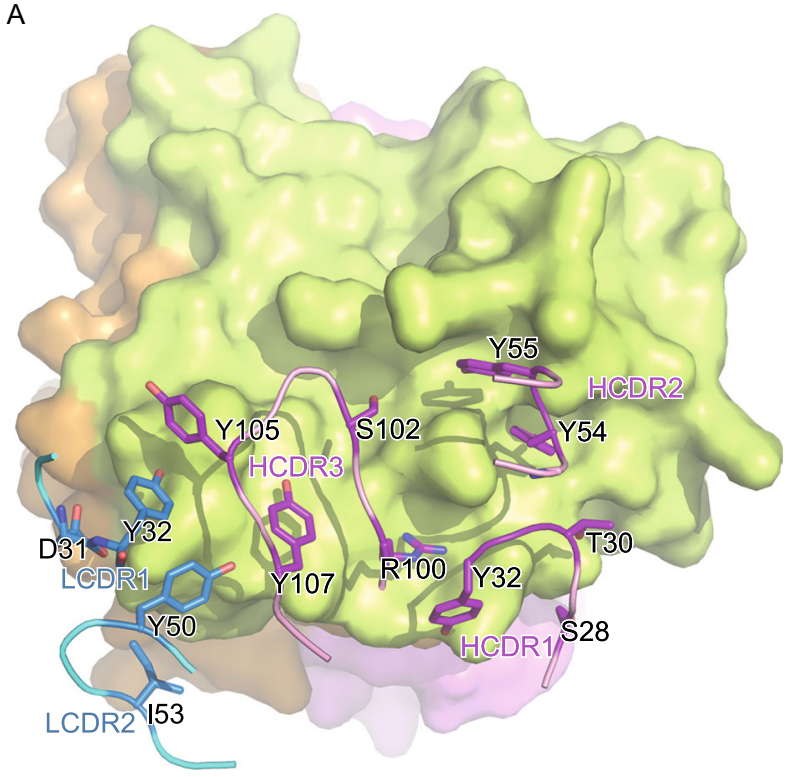

B

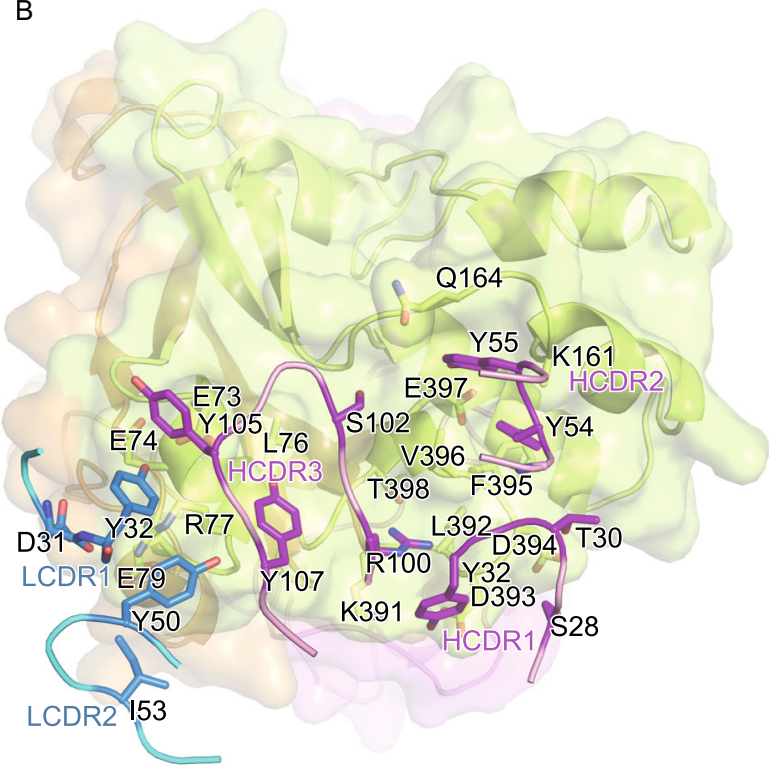

Figure 5. The analysis of the detailed interactions between the JL2 Fab and SCARB2. (A) Five polypeptide elements, three from the heavy chain (purple) and two from the light chain (marine), surround the domain III of SCARB2. Ten residues, S28, T30, G31, Y32, Y54, Y55, R100, S102, Y105, and Y107, in the heavy chain and four residues, D31, Y32, Y50, and I55, in the light chain interact with SCARB2 (with distance less than $4.5 \AA$ ). The residues in JL2 Fab involved in the interactions with SCARB2 are shown as sticks. (B) Similar to (A), a semi-transparent surface rendering of SCARB2 presents the detailed interactions. All residues involved in the interactions are shown as sticks.

Table 2. Interaction residues between JL2 Fab and SCARB2

\begin{tabular}{|c|c|c|c|}
\hline & Antibody & Distance $(\AA)$ & SCARB2 \\
\hline \multirow[t]{10}{*}{$\mathrm{H}$ chain } & S28 & 3.90 & D394 \\
\hline & T30 & 4.33 & V396 \\
\hline & G31 & 3.82 & V396 \\
\hline & Y32 & $2.94,4.52$ & K391, D393 \\
\hline & Y54 & $\begin{array}{l}3.33,3.35,3.28 \\
3.77,3.67\end{array}$ & $\begin{array}{l}\text { K161, D394, F395, } \\
\text { V396, E397 }\end{array}$ \\
\hline & Y55 & $3.72,4.04,3.27$ & Q164, V396, E397 \\
\hline & $\mathrm{R} 100$ & $\begin{array}{l}2.33,3.81,2.33 \\
2.31\end{array}$ & $\begin{array}{l}\text { L392, D393, T398, } \\
\text { G399 }\end{array}$ \\
\hline & S102 & 4.45 & Q164 \\
\hline & Y105 & 3.68 & E73 \\
\hline & Y107 & $3.99,2.88,4.33$ & E73, L76, R77 \\
\hline \multirow[t]{4}{*}{$L$ chain } & D31 & 4.14 & $\mathrm{R} 77$ \\
\hline & Y32 & $4.20,4.50,3.81$ & $\mathrm{E} 73, \mathrm{E} 74, \mathrm{R} 77$ \\
\hline & Y50 & 3.81 & $\mathrm{R} 77$ \\
\hline & 155 & 4.36 & E79 \\
\hline
\end{tabular}

Interaction residues between JL2 Fab and SCARB2 were identified by observing pairs of side chain atoms a separation of $<4.5 \AA$.

aa of mouse SCARB2 in this region with the counterpart sequence from human SCARB2 confers EV71 infectivity (Chen et al., 2012). We showed that JL2 weakly binds to chimeric SCARB2 using the residues 144-151 and 302478 from the human sequence (Figs. $3 C$ and $4 D$ ). It is worth pointing out that JL2 does not bind to 144-151 aa directly (Table 1); thus, the species-specific amino acids in those two regions may affect the overall protein folding and consequently affect the structure of the JL2 binding site. 
Collectively, there are at least two possible ways for JL2 to block EV71 infection: 1) JL2 competes with EV71 for SCARB2 binding or 2) JL2 may stabilize the SCARB2 structure at neutral $\mathrm{pH}$ and prevent EV71 uncoating. The exact mechanism of how JL2 blocks EV71 infection needs to be further elucidated after the EV71-SCARB2 complex structure is determined.

\section{MATERIALS AND METHODS}

\section{Cells and viruses}

The human embryonic kidney (HEK) 293T, 293A, SP2/0, and L929 cell lines were purchased from ATCC. 293A is a subclone of the human embryonic kidney (HEK) 293 cell line and has a relatively flat morphology. L929 is a mouse fibroblastic cell line. The 293-hSCARB2 cell line, which stably expresses human SCARB2 on the cell surface, was established in our lab. The 293-SCARB2-KO cell line, which does not express human SCARB2 because the gene encoding SCARB2 protein was knocked out using the CRISPRCAS9 technique, was established in our lab. 293A, 293-hSCARB2, 293T, 293-SCARB2-KO, and L929 cells were maintained in Dulbecco's modified Eagle's medium (DMEM) (Invitrogen) containing $10 \%$ fetal bovine serum (FBS) (HyClone) and $1 \%$ penicillin/streptomycin at $37^{\circ} \mathrm{C}$ in a $5 \% \mathrm{CO}_{2}$ incubator. SP2/0 cells were grown in RPMI 1640 medium (Invitrogen) with 10\% FBS and 1\% penicillin/ streptomycin at $37^{\circ} \mathrm{C}$ in a $5 \% \mathrm{CO}_{2}$ incubator.

Sf9 cells were cultured in Serum-Free Medium at $27^{\circ} \mathrm{C}$ on a shaker at $100 \mathrm{rpm}$. The construct of the ectodomain of human SCARB2 (residues from 37 to 429) was inserted into the pFastBac_Bee vector for transformation into Sf9 cells. The pFastBac_Bee is a vector that has been modified from pFastBac-one and can express recombinant proteins with a melittin tag at the $\mathrm{N}$-terminus and a $6 \times$ His-tag at the C-terminus.

Enterovirus 71 (EV71, 0804232Y) was kindly provided by Dr. Honglin $\mathrm{Xu}$ from the National Vaccine and Serum Institute. EV71-GFP virus was generated by the infectious clone technique (Yamayoshi et al., 2009). Both viruses were propagated in 293-hSCARB2 cells. The cells infected by EV71-GFP virus express GFP and can be visualized under a fluorescence microscope or can be checked by flow cytometry because the GFP will be translated during virus replication.

\section{Antibodies and reagents}

PE goat anti-mouse IgG2a (407108) was purchased from Biolegend. Anti-SCARB2 polyclonal (AF1966) was purchased from R\&D Systems. The mouse anti- $\beta$ actin monoclonal antibody (TA-09), goat anti mouse HRP (ZB-2305), and rabbit anti goat HRP (ZB-2306) were purchased from ZSGB-BIO. FITC-anti human SCARB2 mAb (JL1FITC) were prepared in our lab and labeled by Tianjin Sungene Biotech Co., Ltd. (Tianjin, China).

Production of monoclonal antibodies against human SCARB2

The full-length coding region of the human SCARB2 gene was cloned into the pEGFP-C1 plasmid using PCR. L929 cells in a 60 $\mathrm{mm}$-dish were transfected with plasmids containing the human
SCARB2 gene. $48 \mathrm{~h}$ later, the hSCARB2-transfected L929 cells, together with $10 \mu \mathrm{g}$ of mouse CpG (ODN1826, Invitrogen), were used to immunize 10-week-old BALB/c female mice via hypodermic injection. The mice were boosted three times at one-month intervals. Three days after the final boost immunization (without $\mathrm{CpG}$ ), mouse splenocytes were taken out and fused to SP2/0 myeloma cells using $50 \%$ polyethylene glycol (PEG) 4000 (Sigma-Aldrich). The cells were seeded into 96-well plates containing Hypoxanthine/Aminopterin/Thymidine (HAT) (Sigma-Aldrich) medium, which were seeded with feeder cells (peritoneal macrophages) $24 \mathrm{~h}$ before. The hybridomas' culture supernatants were screened by flow cytometry with human SCARB2-transfected 293T cells using a Guava easyCyte $^{\mathrm{TM}}$ device (Merck Millipore). The positive clones were subcloned for 4 rounds again until the positive rate was $100 \%$, and then, the monoclonal hybridomas were frozen and stored in liquid nitrogen.

Ascites was prepared via an intraperitoneally injection into pristane-treated $\mathrm{Rag}^{-/-} \mathrm{Gamma}^{-/-}$Caspase $8^{+}$mice with $1 \times 10^{6}$ hybridoma cells per mouse. The mAb was purified from ascites using Protein G Sepharose 4 Fast Flow beads (GE Healthcare). The IgG isotype was determined by an ELISA and flow cytometry using the anti-mouse $\lg G 1, \lg G 2 a, \lg G 2 b, \lg G 3, \lg K$, and $\lg \lambda$ (Biolegend).

The binding efficiency of human SCARB2 and the anti-human SCARB2 mAb

To test the efficiency of JL2 binding to human SCARB2, the JL2 mAb and isotype control $\mathrm{mAb}$ were diluted in complete DMEM at the indicated concentration. 293-hSCARB2 cells were digested with 2 $\mathrm{mmol} / \mathrm{L}$ EDTA, and aliquoted. The diluted $\mathrm{mAbs}$ were added to each aliquot of cells and incubated on ice for $30 \mathrm{~min}$. After washing, 1:1000-diluted PE-labeled goat anti mouse IgG2a was added to each aliquot of cells, and the cells were incubated on ice for $30 \mathrm{~min}$. Then, the cells were washed and fixed with $2 \%$ PFA. The cells were analyzed by flow cytometry. The median fluorescence intensity (MFI) of $\mathrm{PE}$ for each sample was used to determine the binding efficiency of $\mathrm{mAb}$ to human SCARB2.

\section{mAb blocking experiments}

To assess whether anti-human SCARB2 mAb can block the EV71 virus infection of 293-hSCARB2 cells, the cells were pretreated with the anti-human SCARB2 mAb for one hour before being exposed to the EV71-GFP virus. Briefly, the cells were seeded in a 24-well plate at $1 \times 10^{5}$ cells per well. During the next day, the anti-human SCARB2 mAb JL2 and the isotype control were diluted at the indicated concentration in complete DMEM. The diluted mAb was added into each well, $200 \mu \mathrm{L} /$ well, and the cells were incubated in an incubator at $37^{\circ} \mathrm{C}$ for $1 \mathrm{~h}$. The EV71-GFP viruses were added directly into each well without removing the mAb. The plate was incubated in an incubator at $37^{\circ} \mathrm{C}$ for 14 or $20 \mathrm{~h}$. The cells were observed under a fluorescence microscope directly or digested with $0.025 \%$ trypsin and fixed with $4 \%$ PFA. Each sample was analyzed with a Guava easyCyte ${ }^{\mathrm{TM}}$ Flow Cytometer (Merck Millipore). GFPpositive cells indicated productive EV71-GFP virus infection.

Additionally, we determined whether JL2 mAb could block wild type EV71 infection. $4 \times 10^{5}$ cells were pretreated with JL2 at $2 \mu \mathrm{g} / \mathrm{mL}$. They incubated in an incubator at $37^{\circ} \mathrm{C}$ for $1 \mathrm{~h}$. Then wild type EV71 at an MOI of 0.1 was added to infect the pretreated cells 
for $1 \mathrm{~h}$. The cells were washed 3 times and resuspended in $2 \mathrm{~mL}$ of complete medium. The cells were seeded into a 24-well plate at $500 \mu \mathrm{L}$ per well in triplicate. The last $500 \mu \mathrm{L}$ were collected as $1 \mathrm{~h}$ samples. The infection supernatants were collected at $18 \mathrm{~h}$ after infection, and the viral RNA was detected by real-time PCR. The virus RNA was extracted by using the Biospin Virus RNA Extraction Kit (Bioflux). The RNA samples were reverse transcribed using the EV71 RT primer, 5'-AATTGTCACCATAAGCAGCCA-3', and M-MLV reverse transcriptase (M1705, Promega) according to the manufacturer's instructions. All the transcripts were quantified using TaqMan real-time PCR with a Rotor-Gene Corbett $65 \mathrm{HO}$ system (Corbett Lifescience). The primers used were as follows (Nijhuis et al., 2002): probe, 5'-CGGAACCGACTACTTTGGGTGTCCGT-3'; forward primer, 5'-TCCTCCGGCCCCTGA-3'; reverse primer, 5'-AA TTGTCACCATAAGCAGCCA-3'.

The data are presented as the fold-increase in the viral load in the $24 \mathrm{~h}$ samples relative to the $1 \mathrm{~h}$ samples.

\section{Establishment of the 293-SCARB2-KO cell line}

We constructed CRISPR-CAS9 plasmids with SCARB2-sgRNA and transferred these plasmids into 293T cells. After $48 \mathrm{~h}$ of transfection, 293 T cells were seeded into 96-well plates to subclone. When the subcloned cells grew to approximately $10^{4}$, half of the cells were used for RNA extraction, and the RNA was reverse transcribed into cDNA using Oligo d:T. Then, PCR was used to amplify the fragments using cDNA as templates and linked the fragments into a T-vector for sequencing. Positive clones were screened for further cloning until the homozygote mutations appeared. Finally, the 293-SCARB2-KO cells were detected by Western blotting.

The sequences of the SCARB2 sgRNAs are as follows: sgRNA1, GCCTCACCTTCTCGATACTC; sgRNA2, GCGTGACGCTGGTCA CCAGC; sgRNA3, TGTAGACCAGAGTATCGAGA.

\section{Mapping of the binding site}

293-SCARB2-KO cells were transfected with vectors that express human and mouse SCARB2 chimeras using Lipofectamine 2000 (Invitrogen). After $36 \mathrm{~h}$, the transfected cells were digested with $2 \mathrm{mmol} / \mathrm{L}$ EDTA. The cells were incubated with JL2 and the isotype control followed by PE-labeled goat anti mouse IgG2a staining and flow cytometry analysis.

\section{SCARB2 expression and purification}

The recombinant SCARB2 protein was produced using Sf9 cells by infecting 1 liter of cells $\left(2 \times 10^{6} / \mathrm{mL}\right.$ confluence) with $10 \mathrm{~mL}$ of P3 viral stock that was obtained following the manufacturer's instructions (Invitrogen). The medium containing the secreted protein was collected after 3-4 days of incubation.

The cell culture medium of the Sf9 cells was harvested and centrifuged for $30 \mathrm{~min}$ at $4000 \times \mathrm{g}$. The supernatant was filtered through a $0.22-\mu \mathrm{m}$ filter and then dialyzed against a 10 -fold excess of PBS $(\mathrm{pH}=7.4)$. This procedure was repeated twice. The sample was then mixed with pre-equilibrated Ni-NTA Agarose (Qiagen) beads at a ratio of $5 \mathrm{~mL}$ of settled beads per liter culture and stirred at $4^{\circ} \mathrm{C}$ for $2 \mathrm{~h}$. The slurry was loaded onto a $15-\mathrm{mL}$ gravity column (Bio-Rad) and washed with a buffer containing $20 \mathrm{mmol} / \mathrm{L}$ Hepes, $\mathrm{pH}$
$7.5,50 \mathrm{mmol} / \mathrm{L} \mathrm{NaCl}$, and $10 \% \mathrm{v} / \mathrm{v}$ glycerol for $20-30$ column volumes. The beads were further washed using a buffer containing 10 $\mathrm{mmol} / \mathrm{L}$ imidazole until no traces of the protein were detected in the flow-through. SCARB2 bound to the column was eluted in a buffer containing $50 \mathrm{mmol} / \mathrm{L}$ imidazole. It was then concentrated (Amicon Ultra-15 30,000 MWCO, Millipore) to approximate $500 \mu \mathrm{L}$ and loaded onto a Superdex G200 gel filtration column (GE Healthcare) equilibrated with wash buffer. The fractions containing the protein were collected, pooled, and further purified by ion exchange chromatography using a Resource $Q$ column (GE Healthcare). Two peaks were observed during elution and the second peak was collected.

Preparation of the Fab fragments of monoclonal antibodies

The anti-SCARB2 monoclonal antibody (mAb) JL2 was produced from specific hybridomas, whose RNAs were extracted, reversetranscripted into cDNA, amplified by PCR, and sequenced. The amino acid sequence of the JL2 light chain $V$ region is CDIVMTQS PATLSVTPGDRVSLSCRASQSISDYLHWYQQKSHESPRLLIKYAS QSISGIPSRFSGSGSGSDFTLSINSVEPEDVGVYYCQNGHSFPFT, and the amino acid sequence of the JL2 heavy chain $V$ region is EVQLQQSGPELEKPGASVKISCMASGYSFTGYNMNWVKQSNGK SLEWIGNIDPYYGDTRYNQKFKDKATLTVDKSSSTAYMQLKSLTS EDSAVYYCARSRGSTSYFYGMDY. JL2 was purified using Protein A affinity chromatography from mouse ascites. The JL2 Fab fragments were prepared using the Pierce FAB Preparation Kit (Thermo Scientific) according to the manufacturer's instructions. They were then dialyzed against $20 \mathrm{mmol} / \mathrm{L}$ acetate $(\mathrm{pH} \mathrm{5.0)}$ at $4^{\circ} \mathrm{C}$, loaded onto a Mono S column (GE Healthcare), and eluted using a 0-500 $\mathrm{mmol} / \mathrm{L} \mathrm{NaCl}$ gradient. The main peak was collected and dialyzed into PBS buffer. The antibody and the Fab concentrations were determined by measuring the absorbance at $280 \mathrm{~nm}$, and the purity was confirmed through SDS-PAGE analysis.

\section{SCARB2-Fab complex preparation}

The purified JL2 Fab fragments were incubated with purified SCARB2 at $4^{\circ} \mathrm{C}$ for $1 \mathrm{~h}$ at a molar ratio of $1: 1$. The mixture was purified further by size-exclusion chromatography on a Superdex S200 GL10/300 column (GE Healthcare) using $20 \mathrm{mmol} / \mathrm{L}$ Hepes, $\mathrm{pH} 7.5$ and $150 \mathrm{mmol} / \mathrm{L} \mathrm{NaCl}$ as the running buffer. Two peaks were observed during elution. The first peak was collected, concentrated to $5 \mathrm{mg} / \mathrm{mL}$, and screened for crystallization.

\section{Crystallization and structure determination}

The crystals of the SCARB2-Fab complex were obtained after 2-3 days in a condition containing $0.2 \mathrm{~mol} / \mathrm{L}$ sodium citrate tribasic dehydrate, $\mathrm{pH} 8.3$ and $20 \%$ w/v polyethylene glycol 3,350. Diffraction data sets for the SCARB2-Fab complex were collected at the beam line BL18U of the Shanghai Synchrotron Radiation Facility in China, with the highest resolution being $3.5 \AA$, belonging to the space group $\mathrm{C} 2$. The data sets were processed and scaled using the HKL2000 package (Otwinowski and Minor, 1997). The initial structure solutions of the SCARB2-Fab complex were obtained by molecular replacement using the program Phaser v2.1 (McCoy et al., 2007) with the crystal structure of SCARB2 (PDB code: 4TW2) 
(Dang et al., 2014) and the mouse Fab structure (Wang et al., 2017) (PDB code: 5WTG) as a search template. Manual model building and refinement were performed using COOT (Emsley and Cowtan, 2004) and PHENIX (Adams et al., 2010) following rigid body refinement, energy minimization, B-factor refinement, and group NCS constraints. The structural figures were drawn using the program PyMOL (DeLano, 2002).

\section{Statistical analysis}

The data are expressed as the means \pm standard deviations. The significance of the variability among the groups was determined by one-way or two-way analysis of variance using GraphPad Prism (version 4.0) software. Differences were considered statistically significant at a $P$ value $<0.05$.

\section{ACCESSION CODES}

The atomic coordinates of SCARB2 in complex with the JL2 Fab have been submitted to the Protein Data Bank with the accession numbers PDB: 5XBM.

\section{ACKNOWLEDGMENTS}

The authors thank Doctor Honglin Xu (National Vaccine and Serum Institute, Beijing, China) for providing the EV71 virus. We also thank Doctor Chunlai Jiang (Jilin University, Changchun, China), Doctor Wenhui Li (National Institute of Biological Sciences, Beijing, China), and Doctor Hanchun Yang (China Agricultural University, Beijing, China) for the indicated providing plasmids.

This work was supported in part by grants from the National Natural Science Foundation of China (Grant Nos. 31670924 and 31570717), the National Basic Research Program (973 Program) (No. 2014CB542800) and the Open Research Project (2015kf04) from the State Key Laboratory of Biological Macromolecules of the Institute of Biophysics at the Chinese Academy of Sciences. X. Wang was supported by the Young Elite Scientist Sponsorship by CAST.

\section{ABBREVIATIONS}

CDR, complementary determining regions; CPE, cytopathic effect; EV71, Entero virus 71; HFMD, hand-foot-and-mouth; HVR, highly variable region; $\mathrm{mAb}$, monoclonal antibody; $\mathrm{MOI}$, multiplicity of infection; SCARB2, scavenger receptor class B member 2.

\section{COMPLIANCE WITH ETHICS GUIDELINES}

Xuyuan Zhang, Pan Yang, Nan Wang, Jialong Zhang, Jingyun Li, Hao Guo, Xiangyun Yin, Zihe Rao, Xiangxi Wang, and Liguo Zhang declare that they have no conflict of interest. This article does not contain any studies with human or animal subjects performed by the any of the authors.

\section{OPEN ACCESS}

This article is distributed under the terms of the Creative Commons Attribution 4.0 International License (http://creativecommons.org/ licenses/by/4.0/), which permits unrestricted use, distribution, and reproduction in any medium, provided you give appropriate credit to the original author(s) and the source, provide a link to the Creative Commons license, and indicate if changes were made.

\section{REFERENCES}

Adams PD, Afonine PV, Bunkoczi G, Chen VB, Davis IW, Echols N, Headd JJ, Hung LW, Kapral GJ, Grosse-Kunstleve RW et al (2010) PHENIX: a comprehensive Python-based system for macromolecular structure solution. Acta Crystallogr Sect D 66:213-221

Canton J, Neculai D, Grinstein S (2013) Scavenger receptors in homeostasis and immunity. Nat Rev Immunol 13:621-634

Chan KP, Goh KT, Chong CY, Teo ES, Lau G, Ling AE (2003) Epidemic hand, foot and mouth disease caused by human enterovirus 71, Singapore. Emerg Infect Dis 9:78-85

Chen P, Song Z, Qi Y, Feng X, Xu N, Sun Y, Wu X, Yao X, Mao Q, Li $X$ et al (2012) Molecular determinants of enterovirus 71 viral entry: cleft around GLN-172 on VP1 protein interacts with variable region on scavenge receptor B 2. J Biol Chem 287:6406-6420

Cho HK, Lee NY, Lee H, Kim HS, Seo JW, Hong YM, Lee SJ, Lee SW, Cheon DS, Hong JY et al (2010) Enterovirus 71-associated hand, foot and mouth diseases with neurologic symptoms, a university hospital experience in Korea, 2009. Korean J Pediatr 53:639-643

Chua KB, Kasri AR (2011) Hand foot and mouth disease due to enterovirus 71 in Malaysia. Virol Sin 26:221-228

Chua KB, Chua BH, Lee CS, Chem YK, Ismail N, Kiyu A, Kumarasamy $V$ (2007) Genetic diversity of enterovirus 71 isolated from cases of hand, foot and mouth disease in the 1997, 2000 and 2005 outbreaks, Peninsular Malaysia. Malays J Pathol 29:69-78

Dang M, Wang X, Wang Q, Wang Y, Lin J, Sun Y, Li X, Zhang L, Lou Z, Wang $J$ et al (2014) Molecular mechanism of SCARB2-mediated attachment and uncoating of EV71. Protein Cell 5:692-703

DeLano, W.L. (2002). The PyMOL molecular graphics system.

Emsley P, Cowtan K (2004) Coot: model-building tools for molecular graphics. Acta Crystallogr Sect D 60:2126-2132

Fan X, Jiang J, Liu Y, Huang X, Wang P, Liu L, Wang J, Chen W, Wu W, Xu B (2013) Detection of human enterovirus 71 and Coxsackievirus $A 16$ in an outbreak of hand, foot, and mouth disease in Henan Province, China in 2009. Virus Genes 46:1-9

Fujii K, Nagata N, Sato Y, Ong KC, Wong KT, Yamayoshi S, Shimanuki M, Shitara H, Taya C, Koike S (2013) Transgenic mouse model for the study of enterovirus 71 neuropathogenesis. Proc Natl Acad Sci USA 110:14753-14758

Gao M, Duan H, Liu J, Zhang H, Wang X, Zhu M, Guo J, Zhao Z, Meng L, Peng $Y$ (2014) The multi-targeted kinase inhibitor sorafenib inhibits enterovirus 71 replication by regulating IRESdependent translation of viral proteins. Antivir Res 106:80-85

Ho M, Chen ER, Hsu KH, Twu SJ, Chen KT, Tsai SF, Wang JR, Shih SR (1999) An epidemic of enterovirus 71 infection in Taiwan. Taiwan Enterovirus Epidemic Working Group. N Engl J Med 341:929-935 
Hosoya M, Kawasaki Y, Sato M, Honzumi K, Kato A, Hiroshima T, Ishiko $H$, Suzuki $H$ (2006) Genetic diversity of enterovirus 71 associated with hand, foot and mouth disease epidemics in Japan from 1983 to 2003. Pediatr Infect Dis J 25:691-694

Kim SJ, Kim JH, Kang JH, Kim DS, Kim KH, Kim KH, Kim YH, Chung JY, Bin JH, Jung DE et al (2013) Risk factors for neurologic complications of hand, foot and mouth disease in the Republic of Korea, 2009. J Korean Med Sci 28:120-127

Lee KY, Lee MS, Kim DB (2016) Neurologic manifestations of enterovirus 71 infection in Korea. J Korean Med Sci 31:561-567

Lin YW, Lin HY, Tsou YL, Chitra E, Hsiao KN, Shao HY, Liu CC, Sia C, Chong P, Chow YH (2012) Human SCARB2-mediated entry and endocytosis of EV71. PLoS ONE 7:e30507

Lin YW, Yu SL, Shao HY, Lin HY, Liu CC, Hsiao KN, Chitra E, Tsou YL, Chang HW, Sia C et al (2013) Human SCARB2 transgenic mice as an infectious animal model for enterovirus 71. PLoS ONE 8:e57591

Liu MY, Liu W, Luo J, Liu Y, Zhu Y, Berman H, Wu J (2011) Characterization of an outbreak of hand, foot, and mouth disease in Nanchang, China in 2010. PLoS ONE 6:e25287

Matthews BW (1968) Solvent content of protein crystals. J Mol Biol 33:491-497

McCoy AJ, Grosse-Kunstleve RW, Adams PD, Winn MD, Storoni LC, Read RJ (2007) Phaser crystallographic software. J Appl Crystallogr 40:658-674

McMinn PC (2002) An overview of the evolution of enterovirus 71 and its clinical and public health significance. FEMS Microbiol Rev 26:91-107

Mizuta K, Aoki Y, Matoba Y, Yahagi K, Itagaki T, Katsushima F, Katsushima Y, Ito S, Hongo S, Matsuzaki Y (2014) Molecular epidemiology of enterovirus 71 strains isolated from children in Yamagata, Japan, between 1990 and 2013. J Med Microbiol 63:1356-1362

Neculai D, Schwake M, Ravichandran M, Zunke F, Collins RF, Peters J, Neculai M, Plumb J, Loppnau P, Pizarro JC et al (2013) Structure of LIMP-2 provides functional insights with implications for SR-BI and CD36. Nature 504:172-176

Nijhuis M, van Maarseveen N, Schuurman R, Verkuijlen S, de Vos M, Hendriksen K, van Loon AM (2002) Rapid and sensitive routine detection of all members of the genus enterovirus in different clinical specimens by real-time PCR. J Clin Microbiol 40:3666-3670

Ooi MH, Wong SC, Podin Y, Akin W, del Sel S, Mohan A, Chieng $\mathrm{CH}$, Perera D, Clear D, Wong D et al (2007) Human enterovirus 71 disease in Sarawak, Malaysia: a prospective clinical, virological, and molecular epidemiological study. Clin Infect Dis 44:646656

Ooi MH, Wong SC, Lewthwaite P, Cardosa MJ, Solomon T (2010) Clinical features, diagnosis, and management of enterovirus 71 . Lancet Neurol 9:1097-1105

Otwinowski Z, Minor W (1997) Processing of X-ray diffraction data collected in oscillation mode. Methods Enzymol 276:307-326

Sanders SA, Herrero LJ, McPhie K, Chow SS, Craig ME, Dwyer DE, Rawlinson W, McMinn PC (2006) Molecular epidemiology of enterovirus 71 over two decades in an Australian urban community. Arch Virol 151:1003-1013
Sato C, Syoji M, Ueki Y, Sato Y, Okimura Y, Saito N, Kikuchi N, Yagi T, Numakura $H$ (2006) Isolation of enterovirus 71 from patients with hand, foot and mouth disease in a local epidemic on March 2006, in Miyagi prefecture, Japan. Jpn J Infect Dis 59:348

Vega MA, Rodriguez F, Segui B, Cales C, Alcalde J, Sandoval IV (1991a) Targeting of lysosomal integral membrane protein LIMP II. The tyrosine-lacking carboxyl cytoplasmic tail of LIMP II is sufficient for direct targeting to lysosomes. J Biol Chem 266:16269-16272

Vega MA, Segui-Real B, Garcia JA, Cales C, Rodriguez F, Vanderkerckhove J, Sandoval IV (1991b) Cloning, sequencing, and expression of a cDNA encoding rat LIMP II, a novel 74-kDa lysosomal membrane protein related to the surface adhesion protein CD36. J Biol Chem 266:16818-16824

Wang Y, Zou G, Xia A, Wang X, Cai J, Gao Q, Yuan S, He G, Zhang $S$, Zeng $M$ et al (2015) Enterovirus 71 infection in children with hand, foot, and mouth disease in Shanghai, China: epidemiology, clinical feature and diagnosis. Virol J 12:83

Wang X, Zhu L, Dang M, Hu Z, Gao Q, Yuan S, Sun Y, Zhang B, Ren $\mathrm{J}$, Kotecha A et al (2017) Potent neutralization of hepatitis A virus reveals a receptor mimic mechanism and the receptor recognition site. Proc Natl Acad Sci USA 114:770-775

Wu Y, Yeo A, Phoon MC, Tan EL, Poh CL, Quak SH, Chow VT (2010) The largest outbreak of hand; foot and mouth disease in Singapore in 2008: the role of enterovirus 71 and coxsackievirus A strains. Int J Infect Dis 14:e1076-1081

Yamayoshi S, Koike S (2011) Identification of a human SCARB2 region that is important for enterovirus 71 binding and infection. $\mathrm{J}$ Virol 85:4937-4946

Yamayoshi S, Yamashita Y, Li J, Hanagata N, Minowa T, Takemura T, Koike $S$ (2009) Scavenger receptor B2 is a cellular receptor for enterovirus 71. Nat Med 15:798-801

Yamayoshi S, Fujii K, Koike S (2012) Scavenger receptor b2 as a receptor for hand, foot, and mouth disease and severe neurological diseases. Front Microbiol 3:32

Yamayoshi S, Ohka S, Fujii K, Koike S (2013) Functional comparison of SCARB2 and PSGL1 as receptors for enterovirus 71. J Virol 87:3335-3347

Yamayoshi S, Fujii K, Koike S (2014) Receptors for enterovirus 71. Emerg Microbes Infect 3:e53

Zhang Q, MacDonald NE, Smith JC, Cai K, Yu H, Li H, Lei C (2014) Severe enterovirus type 71 nervous system infections in children in the Shanghai region of China: clinical manifestations and implications for prevention and care. Pediatr Infect Dis J 33:482487

Zhou X, Fan G, Xia W, He F, Hu M, Ni X, Zhang Y, Chen H (2013) Molecular epidemiology of human enterovirus 71 strains in the Nanchang region of China in 2011. Jpn J Infect Dis 66:149-150

Zhou S, Liu Q, Wu X, Chen P, Wu X, Guo Y, Liu S, Liang Z, Fan C, Wang $Y$ (2016) A safe and sensitive enterovirus A71 infection model based on human SCARB2 knock-in mice. Vaccine 34:2729-2736

Zou XN, Zhang XZ, Wang B, Qiu YT (2012) Etiologic and epidemiologic analysis of hand, foot, and mouth disease in Guangzhou city: a review of 4,753 cases. Braz J Infect Dis 16:457-465 